\title{
Die Linzer Homöopathieambulanz
}

\author{
Homöopathie ist mit Schulmedizin gut kombinierbar
}

Komplementärmedizin liegt im Trend auch in Oberösterreich. Im Konventhospital der Barmherzigen Brüder in Linz, an der geburtshilflich-gynäkologischen Abteilung unter der Leitung von Prim. Dr. Harald Gründling, können die Patientinnen bereits seit vier Jahren eine begleitende homöopathische Behandlung in Anspruch nehmen. Die homöopathische Ambulanz wird von der Linzer Ärztin Dr. Rosemarie BrunnthalerTscherteu geleitet.

PROMED KOMPLEMENTÄR: Was sind die Schwerpunkte der homöopathischen Ambulanz?

BRUNNTHALER: „Gedacht ist die Ambulanz vorwiegend zur Betreuung von Frauen in der Schwangerschaft, bei der Entbindung und im Wochenbett. Natürlich wird die Homöopathie auch für viele andere gynäkologische Indikationen eingesetzt: Zyklusstörungen, prämenstruelles Syndrom, Regelschmerzen, klimakterische Beschwerden, wiederkehrende vaginale Pilzinfektionen, Myome, Zysten; das Spektrum der Ambulanz reicht bis hin zur homöopathischen Begleittherapie von Krebserkrankungen. Ich denke voll Freude an eine Patientin mit Eierstockskrebs, die nun schon zehn Jahre trotz Metastasen überlebt hat! Immer wieder kommen auch Frauen, die sich ein Kind wünschen, aber nicht schwanger werden, wobei unsere Behandlung die Erfüllung dieses Wunsches häufig ermöglicht. Wir begleiten aber auch In-vitro-Fertilisationen homöopathisch, um die Erfolgschancen zu erhöhen."

Wie viele Frauen haben Sie schon behandelt? Wie ist das Feedback?

BrunNTHALER: „Ich habe in den vergangenen vier Jahren rund 450 Patientinnen im Rahmen der Spitalsambulanz homöopathisch behandelt, viele davon mehrmals. Das Feedback war meist sehr positiv. Wir haben nach dem ersten Jahr mit dem Primar eine Verlaufskontrolle durchgeführt: Von den Patientinnen, die eine Rückmeldung abgegeben haben, berichteten 80 Prozent von einer Besserung der Symptome! Eine über 80jährige Patientin wurde beispielsweise so gut von ihren jahrzehnte- lang bestehenden Depressionen befreit, dass sich sogar ihr Hausarzt von der Homöopathie überzeugen ließ. Das freut uns natürlich! Es werden aber nicht nur ambulante Patientinnen betreut (zu einem Selbstbehalt von je EUR 40 pro Sitzung, das heißt pro Stunde), sondern auch stationäre Patientinnen und werdende Mütter im Kreißsaal (beides kostenlos). - Auch bei einigen Entbindungen konnte ich homöopathisch zu einem sanfteren und rascheren Verlauf beitragen, wie besonders nach vorzeitigem Blasensprung, wo die Wehen ja meist nicht spontan einsetzen, oder bei sehr schmerzhaften Wehen."

Wie funktioniert die Zusammenarbeit mit Ärzten, Hebammen und Krankenschwestern? Gibt es da Akzeptanzprobleme?

BrunNThaleR: „Die Zusammenarbeit mit den Kollegen der Abteilung wird immer besser, denn die Erfolge sprechen sich mit derZeitherum. Besonders unterstützt mich Primarius Dr. Harald Gründling, der die Ambulanz ins Leben gerufen hat. Meine Kollegen schicken mir z.B. Frauen mit Schwangerschaftserbrechen, psychischen Problemen in der Schwangerschaft oder Problemen nach der Entbindung, wie z.B. Stillschwierigkeiten. Es kommen auch Frauen, die bereits eine traumatische Geburt erlebt haben und nun Angst vor der bevorstehenden Entbindung haben. Interessant und auch ermutigend ist die Arbeit mit Krebspatientinnen, die durch die homöopathischen Begleittherapien besser auf Chemo- oder Strahlenbehandlung ansprechen, sie besser vertragen, und sich psychisch besser fühlen. Übrigens, was die Akzeptanz betrifft: Auch Krankenschwestern, Hebammen und andere Angestellte des Hauses haben mich schon konsultiert!“

Sie betreiben selbst eine homöopathische Arztpraxis. Was ist der Vorteil einer Ambulanz?

Brunnthaler: „Die Ambulanz ist in das Krankenhaus eingebunden, gut erreichbar und kostengünstig. Sie wird sehr gern von Frauen aufgesucht, die sich sonst eine homöopathische Behandlung nicht leisten könnten - und ich werde häufig darauf an-

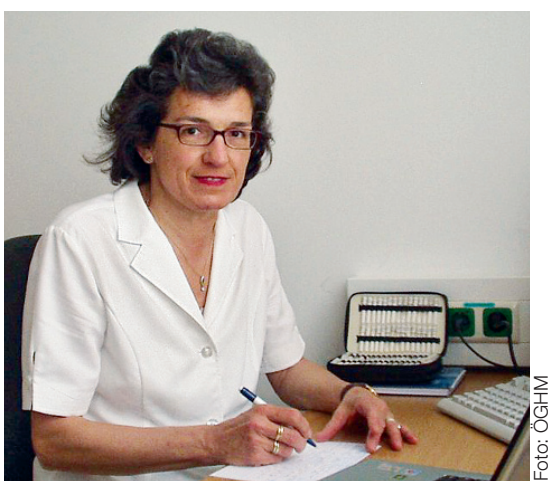

Dr. Rosemarie Brunnthaler-Tscherteu

gesprochen, warum es so etwas nicht auch auf anderen Abteilungen, und besonders für Kinder, gibt. Mir selbst macht es Freude, einmal in der Woche wieder im Rahmen eines Krankenhauses zu arbeiten."

Wie funktioniert die homöopathische Therapie?

BRUNNTHALER: „Im Zentrum der homöopathischen Therapie steht die Arzneimittelfindung im Sinne der klassischen Homöopathie nach Samuel Hahnemann. Das Erstgespräch mit der Patientin dauert rund eine Stunde, spätere Kontrolltermine ca. 30 Minuten. Im Normalfall bekommt die Patientin eine einzige Arznei verordnet, die meist in Form von Globuli oder Tropfen verabreicht wird. Hochpotenzen erhalten die Patientinnen gleich von mir, für die übrigen Potenzen stelle ich Rezepte aus, wobei die hauseigene Apotheke viele Arzneien, die ich häufiger verschreibe, lagernd hat."

Welche Vorteile hat die homöopathische Therapie?

BrunNThaleR: „Ein großer Vorteil ist die nachhaltige Wirkung der Homöopathie, die Abwehrkräfte und Immunsystem langfristig stärkt. Die homöopathischen Arzneien beeinflussen nicht nur den Körper, sondern auch die Seele positiv.

Die Medikamente sind einfach zu handhaben, die Arzneien sind gut verträglich und kostengünstig. Die Homöopathie ist mit der Schulmedizin auch gut kombinierbar und kommt den Wünschen der Patienten nach Zusatztherapie sehr entgegen." 